\title{
Clinical Manifestation and Prognosis of Vestibular Migraine According to the Vestibular Function Test Results
}

\author{
Jae-Wook Lee ${ }^{1}$, Jae Yun Jung ${ }^{1}$, You Sun Chung ${ }^{2}$ and Myung-Whan Suh ${ }^{3}$ \\ ${ }^{1}$ Department of Otolaryngology-Head \& Neck Surgery, Dankook University College of Medicine, Cheonan, \\ ${ }^{2}$ Department of Otorhinolaryngology, Dongguk University College of Medicine, Gyeongju, \\ ${ }^{3}$ Department of Otorhinolaryngology, Seoul National University Hospital, Seoul, Korea
}

\author{
Received November 27, 2012 \\ Revised March 10, 2013 \\ Accepted March 15, 2013
}

Background and Objectives: According to previous reports, patients with vestibular migraine (VM) display variable results from vestibular function tests (VFT): central, peripheral, or normal. The aim of this study was to classify the VM patients into the three groups according to interictal VFT findings (central, peripheral or normal) and to clarify the relationship between VFT results and the clinical manifestations and prognosis in each group. Subjects and Methods: We reviewed the medical records of 81 patients diagnosed as VM using the criteria of Neuhauser, et al. between December 2004 and June 2009. Patients were divided into three groups according to the results of VFT. We compared the clinical manifestations and prognosis between groups. Characteristics including dizziness, the nature of headache, associated otologic symptoms, hearing threshold, duration of illness, and recovery time were analyzed. Results: The number of patients with central, peripheral vestibular dysfunction and normal finding in VFT were 15, 28, and 38 respectively. There were no significant differences in the nature of headache, associated otologic symptoms, hearing threshold, duration of illness, and recovery time. A small difference was observed in the mean age and characteristics of dizziness, but these were not significant. Conclusions: In patients with VM, classification according to the type of vestibular dysfunction was not helpful in the prediction of prognosis and clinical manifestations.

Korean J Audiol 2013;17:18-22

\section{Introduction}

Vestibular migraine (VM) is the second most common cause of recurrent vertigo after benign paroxysmal positional vertigo, and the awareness of migraine as a major cause of dizziness is growing. ${ }^{2)}$ In 2001, Neuhauser, et al. ${ }^{3)}$ formulated diagnostic criteria for VM that were recently validated by the same group. ${ }^{4)}$ They provide a specific category of definite VM and a tentative category of probable VM for patients who do not exhibit all features that VM typically manifests initially. ${ }^{3,4)}$ Using these criteria, VM was identified in $7 \%$ of patients referred to a dizziness clinic and in 9\% referred to a migraine clinic. ${ }^{3)}$ In a Korean multicenter study, VM was identified in $8.5 \%$ of patients who visited the dizziness clinic. ${ }^{5)}$

The pathophysiology of VM is not well-understood. ${ }^{6}$ Various hypotheses are derived from the presumed pathophysiology of migraine. One of these theories suggests that neurogenic inflam- mation of the trigeminal nerve simultaneously triggers sensitization of the central nervous system and causes repetitive stimulation and hypofunction of the vestibular system in the inner ear. ${ }^{7)}$ In addition, vasospasm of the labyrinthine artery could explain the peripheral vestibular and auditory symptoms. ${ }^{8)}$ According to these theories, the pathologic lesion present in a proportion of patients with VM is considered to be peripheral; namely, it is possible that these patients have abnormal peripheral-type vestibular hypofunction. In other patients, the pathologic lesion may be more central, resulting in central-type vestibular function test findings. ${ }^{9)}$

According to previous reports, patients with VM present various results following vestibular function tests (VFT) including: 1) findings suggestive of central vertigo, 2) unilateral vestibular hypofunction or 3) normal findings. ${ }^{10,11)}$ To our knowledge, few studies have correlated the VFT findings of VM patients with the clinical manifestations and prognosis. The aim of this study 
was to classify VM patients into three groups according to interictal VFT findings (central, peripheral or normal) and to clarify the relationship between the VFT results and the clinical manifestation and prognosis in each group.

\section{Subjects and Methods}

The medical records of 81 patients diagnosed as definite or probable VM using the criteria proposed by Neuhauser, et al. ${ }^{3,4)}$ at the Dizziness Clinic between December 2004 and June 2009 , were reviewed retrospectively. The records included 12 males and 69 females; the average age at the time of presentation was $45.2 \pm 16.6$ years.

VFT results were classified into 1) central vestibular dysfunction, 2) peripheral vestibular dysfunction and 3) normal finding according to the modified criteria of Lempert, et al. ${ }^{9}$ When at least one of the following criteria was fulfilled, central vestibular dysfunction was diagnosed; 1) purely vertical or torsional spontaneous nystagmus or perverted head shaking nystagmus, 2) fixation suppression $<50 \%$ during spontaneous nystagmus recordings, caloric tests, or visual-vestibulo ocular reflex (VVOR), 3) positional nystagmus not fulfilling the criteria for canalolithiasis and cupulolithiasis, and 4) gazeevoked nystagmus, saccadic pursuit or any other ocular motor abnormality. Among the four criteria, only 4) was applied when the patient was younger than 65 years, since ocular motor abnormalities may be present even in normal subjects without definite central pathologies. When the criteria for central vestibular dysfunction were not met and all of the following criteria were fulfilled, peripheral vestibular dysfunction was diagnosed; 1) direction-fixed, predominantly horizontal spontaneous nystagmus and/or pathologic nystagmus ( $>3 \%$ ), and/or caloric canal paresis $>25 \%$, and/or slow harmonic acceleration asymmetry $\left.>15^{\circ} 2\right)>50 \%$ decrease in slow phase velocity of spontaneous nystagmus, caloric test, or VVOR with fixation, 3) semicircular canal paresis contralateral to the direction of nystagmus on head impulsive testing (not checked in some patients), and 4) absence of signs of central nervous system involvement. Patients who did not meet the criteria of central or peripheral vestibular dysfunction and showed normal vestibular function were considered the normal vestibular function group.

Characteristics of dizziness, nature of headache, associated otology symptoms (tinnitus, ear fullness, hearing loss), photophobia, phonophobia, visual aura, precipitating factors (food, smell, menstruation), and family history in each group were analyzed retrospectively. To compare hearing levels among the three groups, pure tone threshold averages of low tone (250 to $500 \mathrm{~Hz}$ ), mid tone (1000 to $2000 \mathrm{~Hz}$ ) and high tone (4000 to
$8000 \mathrm{~Hz}$ ) were compared. Hearing loss was defined as a hearing threshold $>25 \mathrm{~dB}$ HL. The hearing threshold of the affected ear was analyzed in the peripheral vestibular dysfunction group and both ears were analyzed in the central vestibular dysfunction group and the normal vestibular function group. All patients were treated with topiramate $50 \mathrm{mg} /$ day (in two divided doses) and in a small number of cases who were resistant to this medication, flunarizine $10 \mathrm{mg} /$ day (in two divided doses) was prescribed. Prognosis was analyzed in a subgroup of $55 \mathrm{pa}-$ tients who were followed up for 1 month or longer. The results are expressed as means \pm standard deviation. Statistical analysis was performed using SPSS ver. 12.0 (SPSS Software, SPSS Inc., Chicago, IL, USA).

A one-way ANOVA and t-test were used to compare mean age, duration of illness (from the first episode of dizziness to the time of presentation to our dizziness clinic), recovery time (duration from diagnosis/treatment to improvement of subjective dizziness) and the frequency of dizziness attacks. $\chi^{2}$ tests were used to assess the proportion of patients with hearing loss ( $>25$ $\mathrm{dB} H L)$ in the low, mid and high frequency range, the characteristics of dizziness, the nature of headache, the associated otologic symptoms (tinnitus, ear fullness), photophobia, phonophobia, visual aura, precipitating factors (food, smell, menstruation) and family history. Dizziness survival curves were statistically compared using the Kaplan-Meier method. A value of $p<0.05$ was considered to indicate significance.

\section{Results}

Based on the criteria of Lempert, et al.,") normal vestibular function was found in 38 (46.9\%) patients (normal group), followed by peripheral vestibular dysfunction in $28(34.6 \%)$ patients (peripheral group). Central vestibular dysfunction was shown in 15 (18.5\%) patients (central group). Gaze-evoked nystagmus, saccadic pursuit or any other ocular motor abnormality was the most common of the four central vestibular dysfunction criteria, being found in 8 of 15 patients. The mean age of the patients was $38.5 \pm 17.8$ years in the central group, $49.0 \pm$ 17.7 years in the peripheral group and $47.3 \pm 14.5$ years in the normal group. A marginally significant difference in mean age was found between the central and peripheral groups $(p=0.051)$.

In 56 ears of the normal group, low-frequency hearing loss was observed in 11 ears, mid-frequency hearing loss in 13 ears and high-frequency hearing loss in 22 ears. In 21 ears in the peripheral group, low-frequency hearing loss was observed in seven ears, mid-frequency hearing loss in four ears and highfrequency hearing loss in 13 ears. In 26 ears of the central vestibular dysfunction group, low-frequency hearing loss was found in three ears, mid-frequency hearing loss in seven ears 

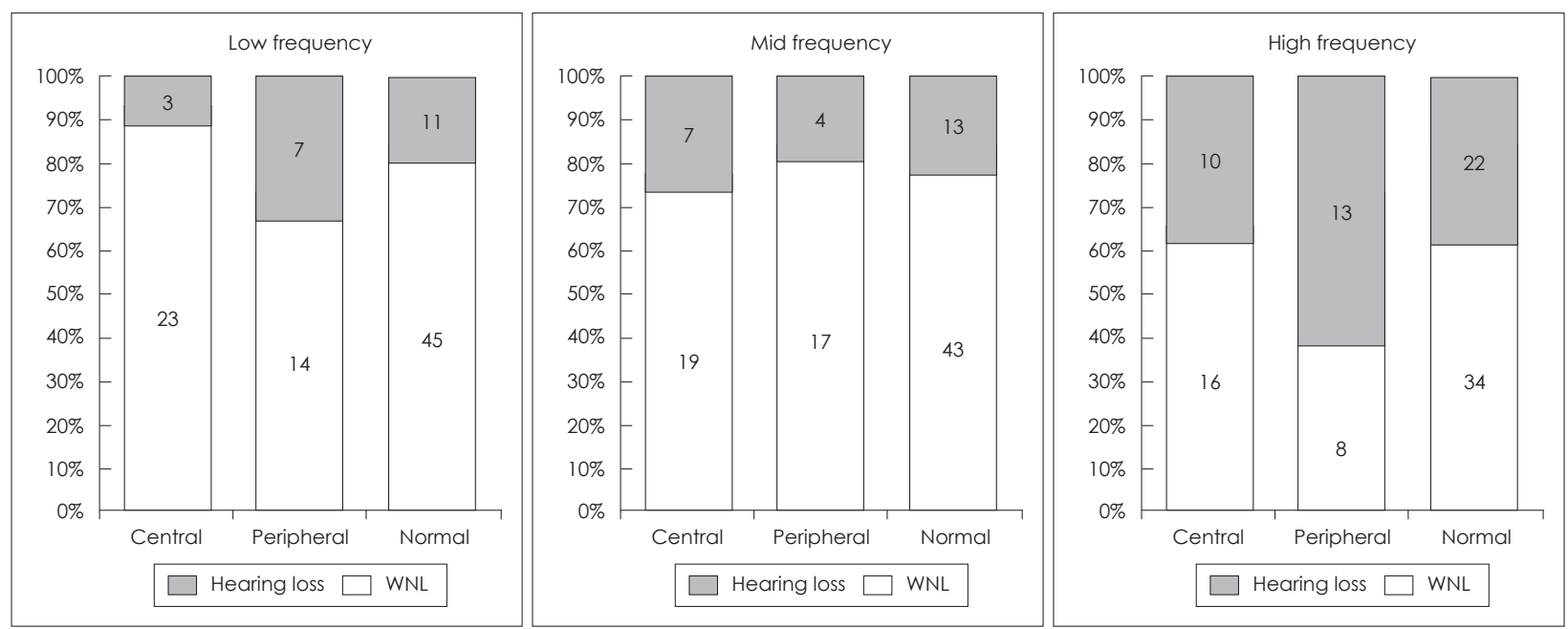

Fig. 1. Incidence of hearing loss. To compare hearing status among the three groups, pure-tone threshold averages of low tone (250 to $500 \mathrm{~Hz})$, mid tone $(1000$ to $2000 \mathrm{~Hz})$ and high tone $(4000$ to $8000 \mathrm{~Hz}$ ) were compared between each group. No significant differences in the proportion of patients with hearing loss $(>25 \mathrm{~dB} \mathrm{HL})$ in the low-, mid- and high-frequency range were observed among the three groups.

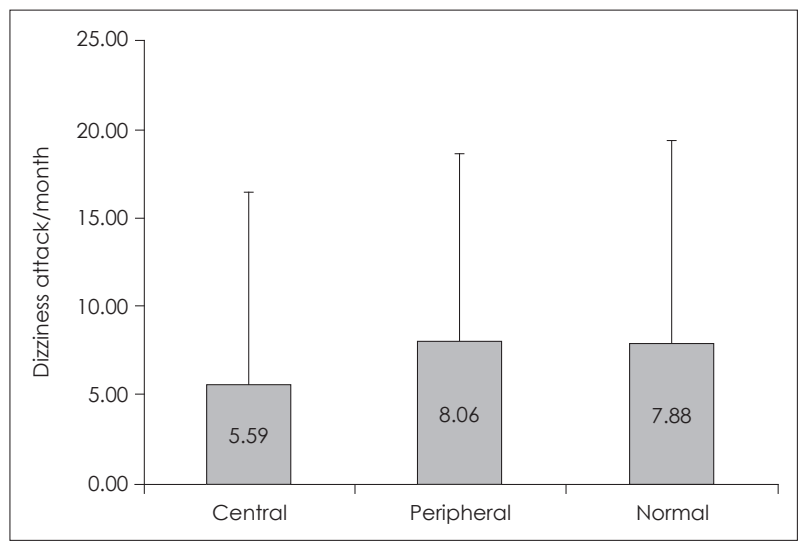

Fig. 2. Frequency of dizziness attacks. The frequencies of dizziness attacks were $7.9 \pm 11.5 /$ month in the normal group, $8.1 \pm 10.6 /$ month in the peripheral group and $5.6 \pm 10.9 /$ month in the central group. No differences among the three groups were identified.

and high-frequency hearing loss in 10 ears. There were no significant differences in the proportion of patients with hearing loss in the low, mid and high frequency range among the three groups (Fig. 1).

The duration of illness (from the first episode of dizziness to the time of presentation to our dizziness clinic) was $7.6 \pm 22.8$ months in the normal group, $15.1 \pm 32.7$ months in the peripheral group and $19.7 \pm 67.2$ months in the central group. There were no differences in the duration of illness among the three groups. The frequencies of dizziness attacks were $7.9 \pm 11.5 /$ months in the normal group, $8.1 \pm 10.6 /$ months in the peripheral group and 5.6 $\pm 10.9 /$ months in the central group. There were no differences in the frequencies of attacks among the three groups (Fig. 2). The characteristics of dizziness were classified as spinning or non-spinning type. Spinning-type dizziness was observed in 19 of $26(73.1 \%)$ patients in the normal group, 11

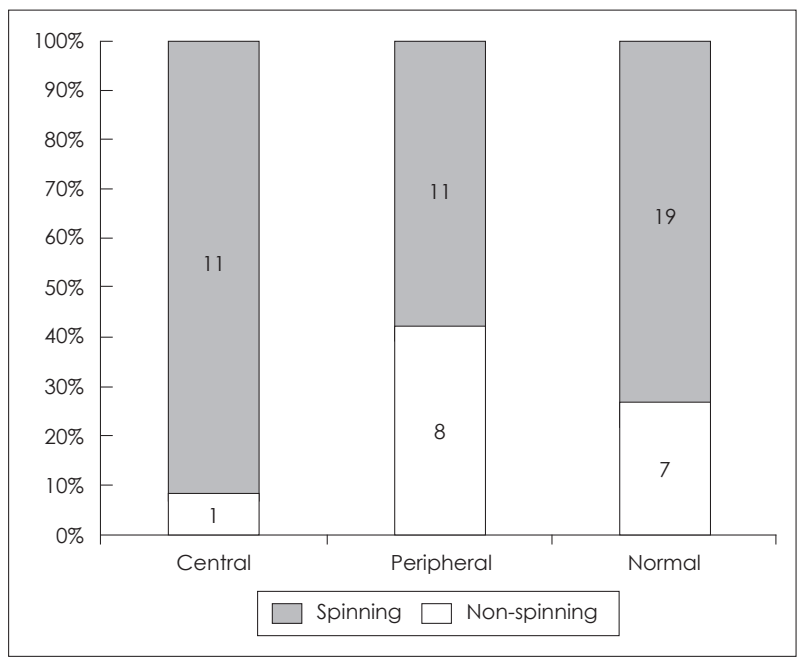

Fig. 3. Characteristics of dizziness. Dizziness was classified as spinning or non-spinning type. Spinning-type dizziness was reported by 19 of 26 (73.1\%) patients in the normal group, 11 of 19 (57.9 $\%)$ in the peripheral group and 11 of $12(91.7 \%)$ in the central group. Spinning-type dizziness occurred more frequently in the central than the peripheral group.

of $19(57.9 \%)$ patients in the peripheral group and 11 of 12 (91.7\%) patients in the central group. Spinning-type dizziness occurred more frequently in the central group than in the peripheral group ( $p=0.050)$ (Fig. 3). Headache was classified as pulsating or non-pulsating; no differences among the three groups were observed.

The recovery times (duration from diagnosis/treatment to improvement of subjective dizziness) were $23.8 \pm 22.3$ days in the normal group, $28.6 \pm 32.0$ days in the peripheral group, and $24.0 \pm 18.3$ days in the central group. The differences were not significant. The Kaplan-Meier curve showed a similar decline in all three groups. Thirty days after diagnosis/treatment, $-60 \%$ 
Fig. 4. Prognosis of dizziness. The recovery time (duration from diagnosis to improvement of subjective dizziness) was $23.8 \pm 22.3$ days in the normal group, $28.6 \pm 32.0$ days in the peripheral group and $24.0 \pm 18.3$ days in the central group (A). These differences were not statistically significant. Kaplan-Meier analysis indicated similar declines in all three groups (B). VFT: vestibular function tests.
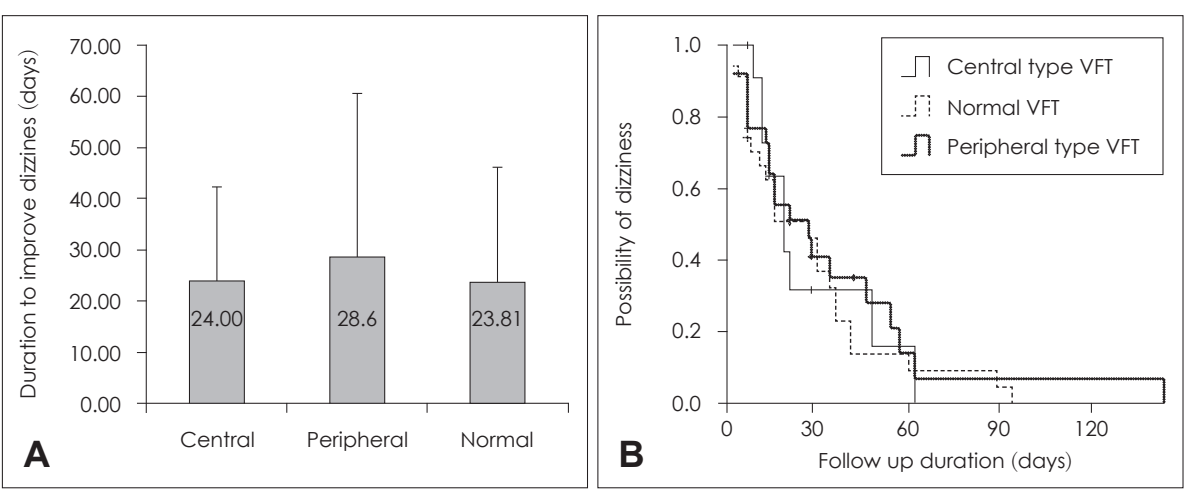

of the patients were symptom free regardless of grouping. After 60 days, $-90 \%$ of the patients were symptom free regardless of grouping (Fig. 4).

When the associated otologic symptoms (tinnitus, ear fullness), photophobia, phonophobia, visual aura, precipitating factors (food, smell, menstruation), and family history were compared among the three groups, no significant differences were found.

\section{Discussion}

Peripheral vestibular dysfunction frequently accompanies atypical VFT results. Although less typical, some central signs may be indicative of central pathology, such as perverted head shake nystagmus ${ }^{12,13)}$ or gazed evoked nystagmus. ${ }^{14,15)}$ These laboratory findings play an important role in localizing the pathology and making a correct diagnosis. A problem during the diagnosis of VM is that all peripheral and/or central laboratory findings may be observed. In this study, $46.9 \%$ of the patients showed normal VFT findings during the interictal period. Approximately $34.6 \%$ showed a peripheral type and $18.5 \%$ showed a central type. As migraine is a disorder of the central nervous system, occurrence of normal- or central-type VFT results is expected. It is important to note that in some patients (34.6\% of our population) the VFT result may resemble a typical unilateral vestibular hypofunction, such as vestibular neuritis. Others have reported VFT results resembling an unilateral vestibular hypofunction in $8-11 \%$ of VM patients during the interictal period. ${ }^{16)}$ In a prospective study of the VFT findings in the ictal period, the incidence of peripheraltype vestibular dysfunction was $15.0 \%{ }^{9}{ }^{9}$ Therefore, VFT results can be variable in VM, and that typical peripheral-type VFT findings cannot completely rule out a diagnosis of VM.

The pathophysiology of VM is still unclear, and several pathophysiological mechanisms that connect migraine-related pathways and the vestibular system have been postulated. ${ }^{10)}$ VM may occur due to the release of neurotransmitters from the inner ear, vasospasm of the spiral modiolar artery, a spread of depression affecting the brainstem or cerebral cortex, genetic defects leading to ion channel dysfunction or abnormal activation of the nucleus of the brainstem. ${ }^{8,17)}$ Stimulation of the trigeminal ganglion may induce significant plasma extravasation from the spiral modiolar artery and the radiating arterioles of the cochlea, causing vertigo, tinnitus, and hearing deficits associated with migraine. ${ }^{7)}$ Vasospasm of the labyrinthine artery could explain the peripheral vestibular and auditory symptoms in migraine. ${ }^{8)}$ According to these theories, the repeated circulation problems and plasma extravasation during migraine attacks may result in permanent damage of the cochlea and vestibule. ${ }^{18-20)}$ That is, in patients with long-standing repeated attacks of migraine, peripheral-type vestibular dysfunction and hearing loss may be more common. In addition, the clinical manifestations of these patients may be similar to typical peripheral vestibular dysfunction.

Against our expectations, we failed to find significant differences among the three groups in terms of clinical manifestations and prognosis. In addition, the hearing results were similar among the three groups. Several explanations for these negative findings are possible: the differences in clinical manifestations may be very subtle, the number of subjects may have been too small, the treatment strategy may have differed between the groups, and VFT may not provide sufficient specificity. The only evidence supporting this hypothesis was that the peripheral group was the oldest (mean age $49.0 \pm 17.7$ ); approximately 10 years older than the central group (mean age $38.5 \pm 17.8$ ), this difference showed marginal significance. It may be that older patients are exposed to a longer duration of repeated migraine attacks, which results in peripheral-type vestibular dysfunction. This may explain why spinning type dizziness occurred more frequently in the central group compared to the peripheral group. That is, whilst the central-type VFT findings may represent current acute dysfunction of the central vestibular system (causing more severe spinning dizziness); peripheral-type VFT findings may represent longstanding chronic dysfunction of the peripheral vestibular system (non-whirling dizziness). Taken together, our results 
do not support the hypothesis that repeated migraines cause permanent damage to the cochlea and vestibule. For instance, no differences in the duration of illness among the three groups was observed. Although VM patients showed variable VFT results, unilateral vestibular dysfunction demonstrated through VFT may not reflect true loss of unilateral vestibular function. Similarly, central vestibular dysfunction demonstrated through VFT may not reflect the true pathology of the central nervous system. Whether such VFT findings are reproducible in the same patients over a particular period of time is unclear. If the VFT findings in VM patients are non-specific, the results may change over time. This issue may be clinically important and requires verification.

Little information is available regarding the treatment of migrainous vertigo. Studies have identified successful treatment using standard migraine therapies of vestibular symptoms in patients with both migraine and vertigo. ${ }^{10)}$ Some studies suggest that drugs used for migraine prophylaxis may be effective. These include propranolol, metoprolol, tricyclic antidepressants, pizotifen, topiramate, and flunarizine. Treatment of acute VM with acute migraine medication can be attempted with triptans and vestibular suppressants such as promethazine, dimenhydrinate, and meclizine. Non-pharmaceutical approaches including the avoidance of identified triggers, stress management and vestibular rehabilitation can be helpful. ${ }^{21)}$ In this study, several medications, including topiramate and flunarizine, were prescribed to the patients. Accordingly, a sophisticated comparison in the clinical manifestation and prognosis was not possible. The results may have been influenced by the medications used. Since the majority of patients were treated with topiramate, and flunarizine was prescribed to few patients, it is unlikely that this factor affected our results.

Although only definite VM patients should be included so as to demonstrate a clear difference in clinical manifestations between groups, we enrolled both definite and probable VM patients in this study. Of our study group, only 45 were definite VM patients. Due to the small sample size, we were unable to find valid differences between groups based on these $45 \mathrm{VM}$ patients. Therefore, 36 probable VM patients were added to the study to increase the sample size, which we deemed important. This may be a limitation of this study.

\section{Conclusion}

We were unable to find clinically significant differences between VM patients with central type VFT results, peripheral type VFT results, and normal VFT results. That is, the clinical manifestations and prognosis were similar in all three groups, regardless of the VFT findings. VFT results in VM patients may be non-specific and clinically non-significant. It therefore appears that the treatment strategy should be focused on the migraine itself rather than the status of vestibular function.

\section{REFERENCES}

1) Neuhauser HK. Epidemiology of vertigo. Curr Opin Neurol 2007; 20:40-6.

2) Rauch SD. Growing awareness of migraine as a major cause of dizziness, imbalance, and vertigo. Introduction. J Vestib Res 2011;21: 297-8.

3) Neuhauser H, Leopold M, von Brevern M, Arnold G, Lempert T. The interrelations of migraine, vertigo, and migrainous vertigo. Neurology 2001;56:436-41.

4) Radtke $A$, Neuhauser $H$, von Brevern M, Hottenrott T, Lempert T. Vestibular migraine--validity of clinical diagnostic criteria. Cephalalgia 2011;31:906-13.

5) Ahn SK, Kang K, Koo JW, Kim KS, Kim BG, Kim BK, et al. Clinical features and treatment pattern of migrainous vertigo in Korea: a nationwide prospective multicenter study. Res Vestib Sci 2009;8: 122-31.

6) Furman JM, Balaban CD, Jacob RG, Marcus DA. Migraine-anxiety related dizziness (MARD): a new disorder? J Neurol Neurosurg Psychiatry 2005;76:1-8.

7) Vass Z, Dai CF, Steyger PS, Jancsó G, Trune DR, Nuttall AL. Co-localization of the vanilloid capsaicin receptor and substance $\mathrm{P}$ in sensory nerve fibers innervating cochlear and vertebro-basilar arteries. Neuroscience 2004;124:919-27.

8) Baloh RW. Neurotology of migraine. Headache 1997;37:615-21.

9) von Brevern M, Zeise D, Neuhauser H, Clarke AH, Lempert T. Acute migrainous vertigo: clinical and oculographic findings. Brain 2005; 128(Pt 2):365-74.

10) Furman JM, Marcus DA, Balaban CD. Migrainous vertigo: development of a pathogenetic model and structured diagnostic interview. Curr Opin Neurol 2003;16:5-13.

11) Johnson GD. Medical management of migraine-related dizziness and vertigo. Laryngoscope 1998;108(1 Pt 2):1-28.

12) Moon IS, Kim JS, Choi KD, Kim MJ, Oh SY, Lee H, et al. Isolated nodular infarction. Stroke 2009;40:487-91.

13) Kim JS, Ahn KW, Moon SY, Choi KD, Park SH, Koo JW. Isolated perverted head-shaking nystagmus in focal cerebellar infarction. Neurology 2005;64:575-6.

14) Strupp M, Hüfner K, Sandmann R, Zwergal A, Dieterich M, Jahn K, et al. Central oculomotor disturbances and nystagmus: a window into the brainstem and cerebellum. Dtsch Arztebl Int 2011;108:197-204.

15) Kattah JC, Talkad AV, Wang DZ, Hsieh YH, Newman-Toker DE. HINTS to diagnose stroke in the acute vestibular syndrome: three-step bedside oculomotor examination more sensitive than early MRI diffusion-weighted imaging. Stroke 2009;40:3504-10.

16) Radtke $A$, von Brevern $M$, Neuhauser $H$, Hottenrott $T$, Lempert $T$. Vestibular migraine: long-term follow-up of clinical symptoms and vestibulo-cochlear findings. Neurology 2012;79:1607-14.

17) Cutrer FM, Baloh RW. Migraine-associated dizziness. Headache 1992;32:300-4.

18) Atkinson M. Migraine and Meniere's disease. Arch Otolaryngol 1962;75:220-5.

19) Rassekh CH, Harker LA. The prevalence of migraine in Menière's disease. Laryngoscope 1992;102:135-8.

20) Parker W. Menière's disease. Etiologic considerations. Arch Otolaryngol Head Neck Surg 1995;121:377-82.

21) Lempert T, Neuhauser H. Epidemiology of vertigo, migraine and vestibular migraine. J Neurol 2009;256:333-8. 